OPEN ACCESS

Edited by:

Peter A. Federolf,

University of Innsbruck, Austria

Reviewed by:

Kaushik Mukherjee,

Indian Institute of Technology Delhi,

India

Chi-Wen Lung,

Asia University, Taiwan

*Correspondence:

S. van Drongelen

stefan.vandrongelen@kgu.de

${ }^{t}$ These authors have contributed equally to this work and share last authorship

Specialty section:

This article was submitted to Biomechanics,

a section of the journal

Frontiers in Bioengineering and

Biotechnology

Received: 10 August 2021

Accepted: 21 October 2021

Published: 04 November 2021

Citation:

van Drongelen S, Braun S, Stief $F$ and Meurer A (2021) Comparison of Gait

Symmetry and Joint Moments in Unilateral and Bilateral Hip

Osteoarthritis Patients and Healthy Controls.

Front. Bioeng. Biotechnol. 9:756460.

doi: 10.3389/fbioe.2021.756460

\section{Comparison of Gait Symmetry and Joint Moments in Unilateral and Bilateral Hip Osteoarthritis Patients and Healthy Controls}

\author{
S. van Drongelen ${ }^{1 *}$, S. Braun ${ }^{2}$, F. Stief ${ }^{2+}$ and A. Meurer ${ }^{2+}$ \\ ${ }^{1}$ Dr. Rolf M. Schwiete Research Unit for Osteoarthritis, Department of Orthopedics (Friedrichsheim), University Hospital Frankfurt, \\ Goethe University Frankfurt, Frankfurt, Germany, ${ }^{2}$ Department of Orthopedics (Friedrichsheim), University Hospital Frankfurt, \\ Goethe University Frankfurt, Frankfurt, Germany
}

Patients with unilateral hip osteoarthritis show a characteristic gait pattern in which they unload the affected leg and overload the unaffected leg. Information on the gait characteristics of patients with bilateral hip osteoarthritis is very limited. The main purposes of this study were to investigate whether the gait pattern of both legs of patients with bilateral hip osteoarthritis deviates from healthy controls and whether bilateral hip osteoarthritis patients show a more symmetrical joint load compared to unilateral hip osteoarthritis patients. In this prospective study, 26 patients with bilateral hip osteoarthritis, 26 patients with unilateral hip osteoarthritis and 26 healthy controls were included. The three groups were matched for gender, age and walking speed. Patients were scheduled for a unilateral total hip arthroplasty on the more affected/more painful side. All participants underwent a three-dimensional gait analysis. Gait kinematics and gait kinetics of patients and controls were compared using Statistical Parametric Mapping. Corrected for speed, the gait kinematics and kinetics of both legs of patients with bilateral hip osteoarthritis differed from healthy controls. Bilateral patients had symmetrical knee joint loading, in contrast to the asymmetrical knee joint loading in unilateral hip osteoarthritis patients. The ipsilateral leg of the bilateral patients could be included in studies in addition to unilateral hip osteoarthritis patients as no differences were found. Although patients with bilateral hip osteoarthritis show more symmetrical frontal plane knee joint moments, a pathological external knee adduction moment in the second half of stance was present in the ipsilateral leg in patients with unilateral and bilateral hip osteoarthritis. The lateral adjustment of the knee adduction moment may initiate or accelerate progression of degenerative changes in the lateral compartment of the knee.

Keywords: hip osteoarthritis (hip OA), unilateral, bilateral, gait symmetry, joint load

Abbreviations: OA, osteoarthritis; THA, total hip arthroplasty; SPM, Statistical Parametric Mapping 


\section{INTRODUCTION}

Patients with unilateral hip osteoarthritis (OA) show a characteristic asymmetric gait pattern in which they unload the affected leg (Schmidt et al., 2017; Wesseling et al., 2018; van Drongelen et al., 2020a). By doing so patients transfer load to the knee and hip joint of the contralateral leg. Schmidt et al. (Schmidt et al., 2017) found that the peak external knee adduction moment in the second half of stance and the peak external hip adduction moment during the first half of stance were respectively 23 and $12 \%$ higher in the contralateral leg. Large cohort studies have shown that unilateral hip OA patients have an increased risk for developing knee OA, in both the ipsilateral but especially in the contralateral knee (Gillam et al., 2013; Jungmann et al., 2015; Joseph et al., 2016). The external knee adduction moment is a commonly used surrogate measure for joint loading and has been related to the initiation and progression of knee OA (Miyazaki et al., 2002; Andriacchi and Mündermann, 2006). Less frequently studied and thus less certain, is the association between the external hip adduction moment and hip OA. Hurwitz et al. (Hurwitz et al., 2001) showed that the initiation of hip OA relates to increased joint loads during walking. Here, the external hip adduction moment has been identified as a major determinant for joint loading (Lenaerts et al., 2009; Wesseling et al., 2015).

To confirm the suspect and define the degree of $\mathrm{OA}$, radiologic images are used. A study of Günther and colleagues (Günther et al., 1998) found radiographic evidence of contralateral hip OA in $82.1 \%$ of patients scheduled for unilateral total hip arthroplasty (THA). Only half of these patients reported pain in the contralateral hip joint. Further, it is known that $16-35 \%$ of patients undergoing THA will receive a contralateral THA within 1 year of the initial hip replacement (Morcos et al., 2018). A recent study from the Osteoarthritis Initiative (Santana et al., 2020) found lower values for the incidence of contralateral THA after the initial THA (8\%) but a clear higher incidence of total knee arthroplasty after an initial THA (32\%).

Studies performing gait analysis predominantly included patients with unilateral hip OA (Shakoor et al., 2011; Allison et al., 2018; Stief et al., 2018; van Drongelen et al., 2021) and as such, studies on the gait characteristics of patients with bilateral hip OA are very limited. Berman et al. (Berman et al., 1991) only studied the spatio-temporal gait parameters. A study from Kubota et al. (Kubota et al., 2007) included kinematics and kinetics, whereas Solomonow-Avnon et al. (SolomonowAvnon et al., 2016) recently reported on a method to reduce the hip joint reaction force by means of footwear. The study from Kubota et al. (Kubota et al., 2007) compared bilateral hip OA patients to healthy controls walking at a free and slow walking speed to eliminate the effect of speed. However, the very low walking speed makes a comparison to the literature difficult. Further, in the study of Kubota et al. (Kubota et al., 2007), no direct comparison to unilateral hip OA patients was made and no knee loads were reported.

Whether bilateral hip OA patients, scheduled for THA surgery on the more affected side, show the characteristic asymmetric gait pattern like unilateral patients or a more symmetrical gait pattern has not been studied yet. The main aim of this study was to determine the gait kinematics, gait kinetics and gait symmetry in patients with bilateral hip OA in comparison to unilateral hip OA patients and healthy controls. It was hypothesized that the gait pattern of both legs of patients with bilateral hip OA deviates from healthy controls, whereas only the affected leg of the unilateral hip OA patients deviates from the healthy control group. We further hypothesized that, in contrast to patients with unilateral hip $\mathrm{OA}$, the external knee and hip adduction moments of the contralateral side are not different compared to the ipsilateral side (more affected side) in bilateral affected patients with hip OA. As a result, bilateral hip OA patients show a more symmetrical joint load compared to unilateral hip OA patients.

\section{METHODS}

\section{Participants}

In this prospective study, data of 26 patients with bilateral hip OA and 26 gender, age and walking speed matched patients with unilateral hip OA were included. All patients were scheduled for a unilateral THA on the more affected/more painful side. Furthermore, 26 healthy controls, also matched for gender, age and walking speed, were selected from our reference database and included for comparison (Table 1). Healthy controls were included if they had no history of orthopedic surgeries, chronic or neuromuscular diseases or recent injuries to the lower extremities. None of the enrolled healthy controls reported ankle, knee, hip or back pain at the time of measurement. All patients and healthy controls gave written informed consent prior to participation in the original studies. Our institution's medical ethics committee approved the studies under the numbers $319 / 11,122 / 14$ and $497 / 15$.

\section{Gait Analysis}

Patients underwent a three-dimensional gait analysis in the week before surgery, whereas the healthy controls visited the gait laboratory within the framework of a reference measurement. The healthy controls were asked to walk at a walking speed of approximately $1.0 \mathrm{~ms}^{-1}$, comparable to patients shortly before surgery (Schmidt et al., 2017). Kinematic data were collected using 8 Vicon MX T10 cameras (VICON Motion Systems, Oxford, United Kingdom) operating at $200 \mathrm{~Hz}$ and two AMTI force plates (Advanced Mechanical Technology Inc., Watertown, MA, United States) were used to synchronously collect ground reaction forces at $1000 \mathrm{~Hz}$. A marker set with markers on the medial malleolus, medial femoral condyle and greater trochanter (Stief et al., 2013) additional to the standardized Plug-in-Gait marker set (Kadaba et al., 1990) was used.

Vicon Nexus (version 2.10) was used to process the marker trajectories. The resulting marker trajectories were filtered using a Woltring filter with a mean squared error setting of 10 (Woltring, 1986). The Plug-in-Gait model (VICON Motion Systems, Oxford, United Kingdom) is Vicon's implementation of the Conventional Gait Model and is still widely used in the clinical gait analysis community (Baker et al., 2018). This 
TABLE 1 | Anthropometric data and walking speed of patients and healthy controls.

\begin{tabular}{|c|c|c|c|c|}
\hline & Bilateral patients & Unilateral patients & Healthy controls & $p$-value \\
\hline Age (years) & $64.5(10.8)$ & $64.1(8.4)$ & $63.3(7.9)$ & 0.885 \\
\hline Height (m) & $1.70(0.09)$ & $1.68(0.09)$ & $1.68(0.10)$ & 0.614 \\
\hline Weight (kg) & $77.4(17.7)$ & $78.4(14.0)$ & $69.3(12.8)$ & 0.063 \\
\hline $\mathrm{BMI}\left(\mathrm{kgm}^{-2}\right)$ & $26.6(4.4)$ & $27.6(4.2)^{\mathrm{a}}$ & $24.6(3.1)$ & 0.026 \\
\hline Speed $\left(\mathrm{ms}^{-1}\right)$ & $0.99(0.13)$ & $1.01(0.13)$ & $1.06(0.13)$ & 0.177 \\
\hline
\end{tabular}

Values are mean values with standard deviation in parenthesis.

BMI: body mass index.

${ }^{a}$ Significantly different compared to healthy controls ( $\mathrm{p}=0.024$ with Bonferroni posthoc test).

model was used to calculate the kinematic and kinetic gait variables. The hip joint centers were obtained according to a geometrical prediction method using regression equations (Davis et al., 1991) whereas the centers of rotation for the ankle and knee joints were defined statically as the midpoint between the medial and lateral malleolus and femoral condyle markers. Kinematic outputs were calculated from the embedded coordinate system information. The joint angles mainly describe the orientation of the distal segment with respect to that of the proximal segment. The orientation of the pelvis is output as segment angles with respect to the laboratory-based axis system, as is the transverse plane alignment of the foot (the so-called foot progression angle). Inverse dynamics were used to estimate joint moments from force plate measurements of the ground reaction, an estimate of segment accelerations from kinematic data and estimates of segment inertial parameters (Kadaba et al., 1990; Davis et al., 1991).

\section{Data Analysis}

For all patients the leg to be operated on (the more affected/more painful side) was considered the ipsilateral leg, whereas the nonaffected side (unilateral patients) and the less affected side (bilateral patients) was called the contralateral leg. For the healthy controls the kinematics and kinetics of the left side were chosen randomly for comparison.

Kinematic and kinetic data were exported to Matlab (version R2020b, The Mathworks Inc., Natick, MA, United States) to normalize the data over the gait cycle and average the parameters over five good trials.

The following kinematic gait parameters, which are typically influenced by hip OA, were included for further analysis: thorax tilt and lean (the lateral displacement of the trunk relative to the supporting limb), pelvic tilt and obliquity, hip flexion-extension, knee flexion-extension, ankle dorsiflexion-plantarflexion and the foot progression angle (the angle of the long axis of the foot segment relative to the direction of walking). External ankle, knee and hip joint moments (expressed in $\mathrm{Nmkg}^{-1}$ ) were defined as kinetic outcomes. In addition, the impulse for the knee adduction and hip adduction moment (area under the curve (Herger et al., 2019)) were calculated.

For the peak external knee and hip adduction moment during the first and second half of the stance phase as well as for the impulse of the adduction moments, an absolute symmetry index
(ASI) was determined (Robinson et al., 1987; Bosch and Rosenbaum, 2010) by the following equation:

$$
A S I=\left|\frac{2\left(X_{L}-X_{R}\right)}{\left(X_{L}+X_{R}\right)}\right| \times 100 \%
$$

$\mathrm{X}_{\mathrm{L}}$ and $\mathrm{X}_{\mathrm{R}}$ are the parameters of the ipsilateral and contralateral leg for patients and the left and right leg for the healthy controls. An ASI of 0 indicates perfect symmetry.

\section{Statistical Analysis}

Shapiro-Wilk tests were used to test for normal distribution of the anthropometrics. Since the data showed a normal distribution, one-way ANOVA (group) with Bonferroni posthoc tests were used to determine statistical differences between the anthropometrics and walking speed of the patient groups and healthy controls.

The selected gait kinematics and kinetics were evaluated using Statistical Parametric Mapping (SPM). SPM originates from the Random Field Theory (Adler and Taylor, 2007) and has been validated for $1 \mathrm{D}$ biomechanical data by Pataky et al. (Pataky et al., 2013). For the kinematics and kinetics, normal distribution was tested and confirmed by SPM. The SPM analyses were performed in MATLAB using the open-source spm1d code (version M.0.4.3) with a general critical threshold ( $p$-value) of $\alpha=0.05$. SPM paired sample t-tests were used to compare the ipsilateral and contralateral legs of the patients. Data of the bilateral, unilateral and healthy controls were compared first with an SPM ANOVA and afterwards with independent two-sample t-tests (critical $p$-value corrected for multiple testing, $\alpha=0.05$ / 3). Data were considered significantly different when the waveform exceeded this critical threshold for more than four successive time points, i.e., at least $4 \%$ of the gait cycle (Wesseling et al., 2018).

Symmetry between patients and healthy controls was tested with Kruskal-Wallis tests and Mann-Whitney tests as the data appeared not be to be normally distributed. $P$-values $\leq 0.05$ were considered significant for all analyses.

\section{RESULTS}

\section{Participants}

In all groups (bilateral patients, unilateral patients and healthy controls), 16 women and 10 men were included. As patients and healthy controls were age and speed matched, no significant 

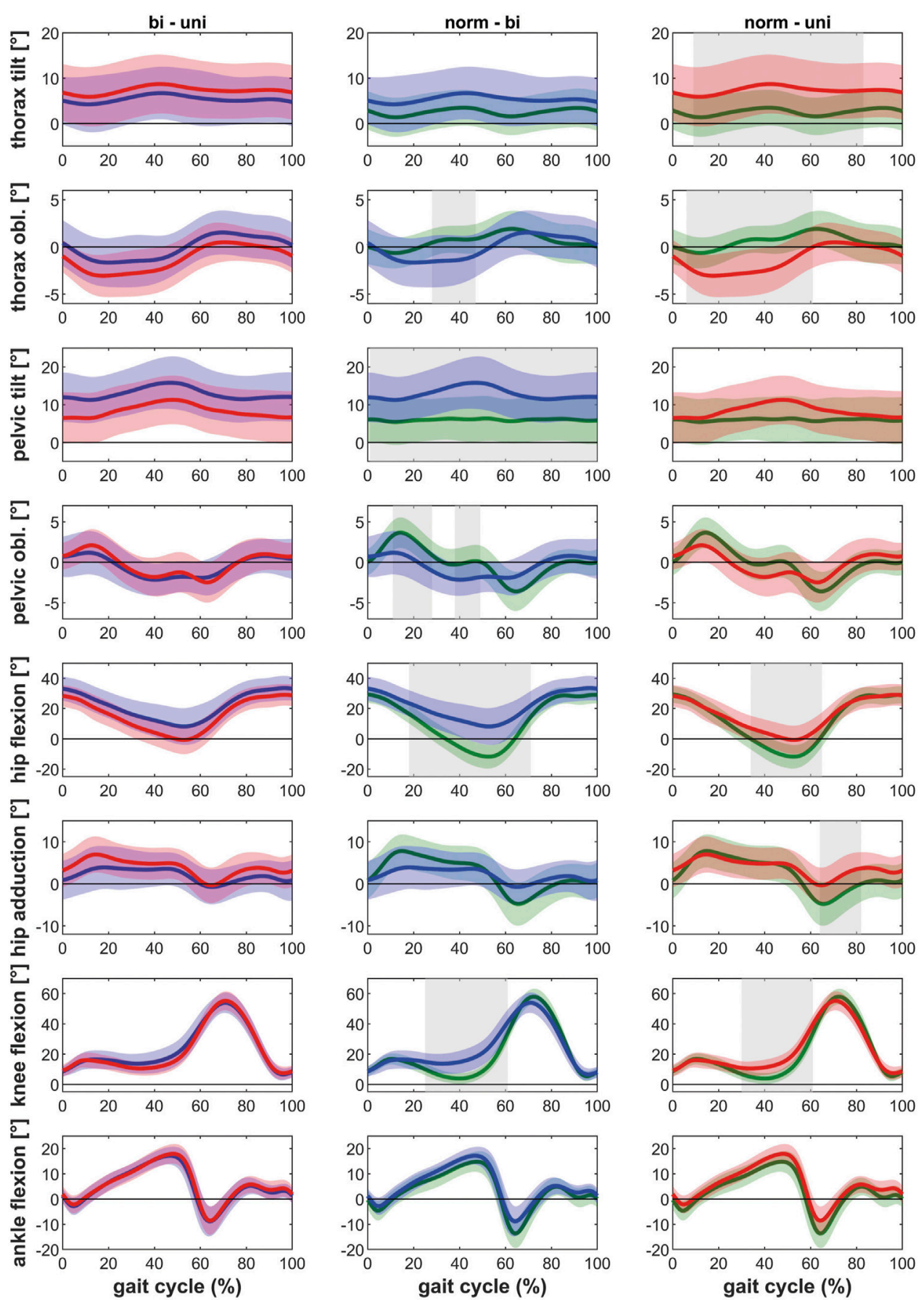

FIGURE 1 | Comparison of the kinematics of the ipsilateral leg between bilateral hip OA patients, unilateral hip OA patients and healthy controls. Mean and one standard deviation of the joint angles. Shaded areas indicate statistical differences. Blue lines $=$ bilateral patients, red lines $=$ unilateral patients, green lines $=$ healthy controls. (A-C) thorax tilt: anterior (+)/posterior (-); (D-F) thorax obliquity: towards the contralateral side (+)/towards the ipsilateral side (-); (G-I) pelvic tilt: anterior (+)/posterior (-); (J-L) pelvic obliquity: up (+)/down (-); (M-O) hip flexion/extension: flexion (+)/extension (-); (P-R) hip adduction/abduction: adduction $(+) / a b d u c t i o n(-)$; (S-U) knee flexion/extension: flexion (+)/extension (-); (V-X) ankle dorsiflexion/plantarflexion: dorsiflexion (+)/plantarflexion (-).

differences were present in age and speed, nor in weight and height (Table 1). Only the Body Mass Index (BMI) was significantly higher in unilateral hip OA patients compared to healthy controls. All bilateral patients had a Kellgren-Lawrence score (Kellgren and Lawrence, 1957) of at least 5 in both legs (Grade 3), whereas the unilateral patients were selected not to have a score exceeding 2 (Grade 1) in the contralateral leg. All patients reported significant pain in the ipsilateral hip joint: 34 

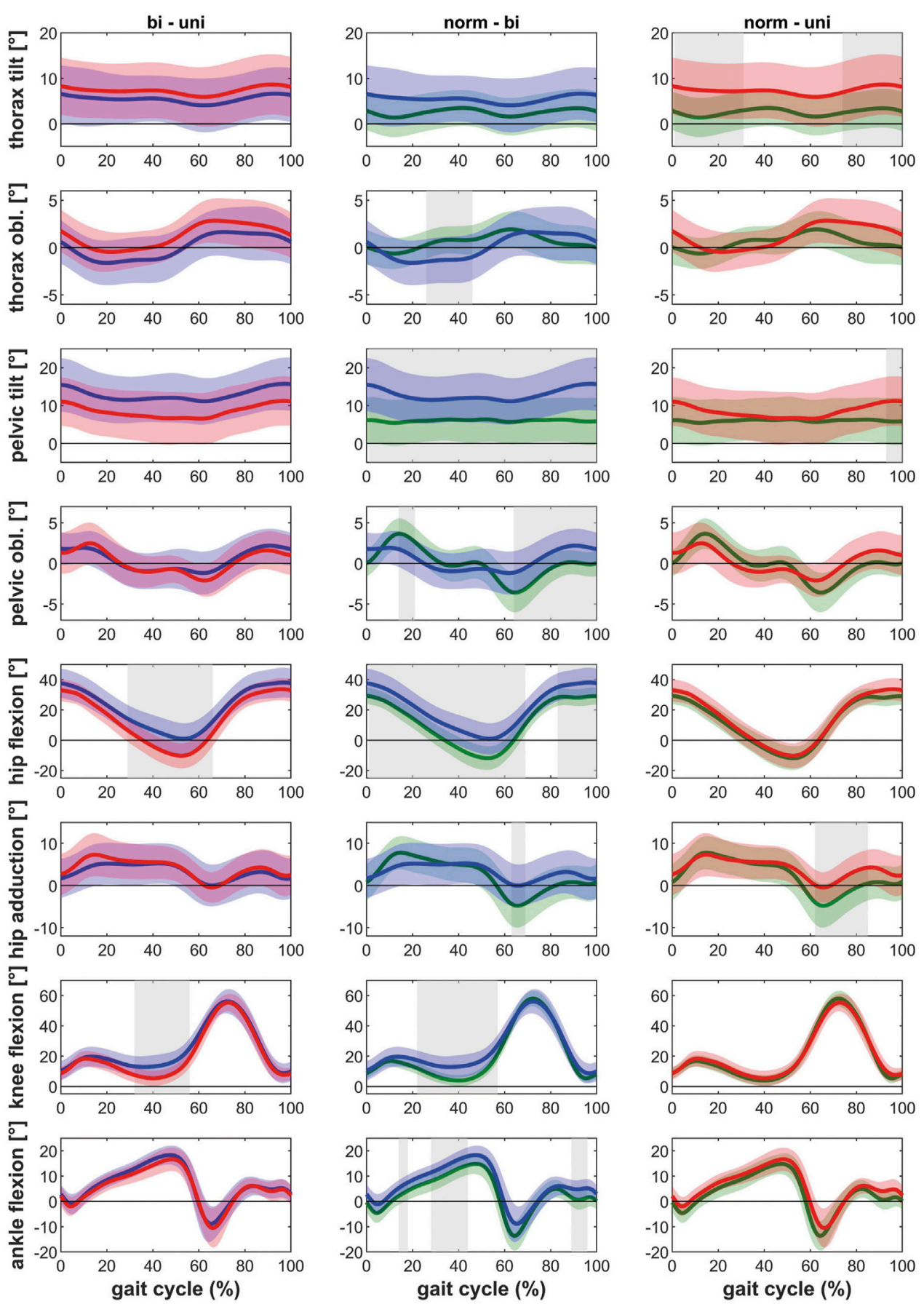

FIGURE 2 | Comparison of the kinematics of the contralateral leg between bilateral hip OA patients, unilateral hip OA patients and healthy controls. Mean and one standard deviation of the joint angles. Shaded areas indicate statistical differences. Blue lines = bilateral patients, red lines = unilateral patients, green lines = healthy controls. (A-C) thorax tilt: anterior (+)/posterior (-); (D-F) thorax obliquity: towards the contralateral side (+)/towards the ipsilateral side (-); (G-I) pelvic tilt: anterior (+)/posterior (-); (J-L) pelvic obliquity: up (+)/down (-); (M-O) hip flexion/extension: flexion (+)/extension (-); (P-R) hip adduction/abduction: adduction $(+) / a b d u c t i o n(-)$; (S-U) knee flexion/extension: flexion (+)/extension (-); (V-X) ankle dorsiflexion/plantarflexion: dorsiflexion (+)/plantarflexion (-).

patients scored 45.9 (11.4) on the pain section of the Hip disability and Osteoarthritis Outcome Score (Nilsdotter et al., 2003; Blasimann et al., 2014), whereas 18 patients from another study scored 55.2 (12.7) on the Harris Hip Score (Harris, 1969).

\section{Kinematics and Kinetics}

In the ANOVA significant differences were detected between the bilateral patients, unilateral patients and healthy controls for both the ipsilateral and contralateral leg for all parameters except for 

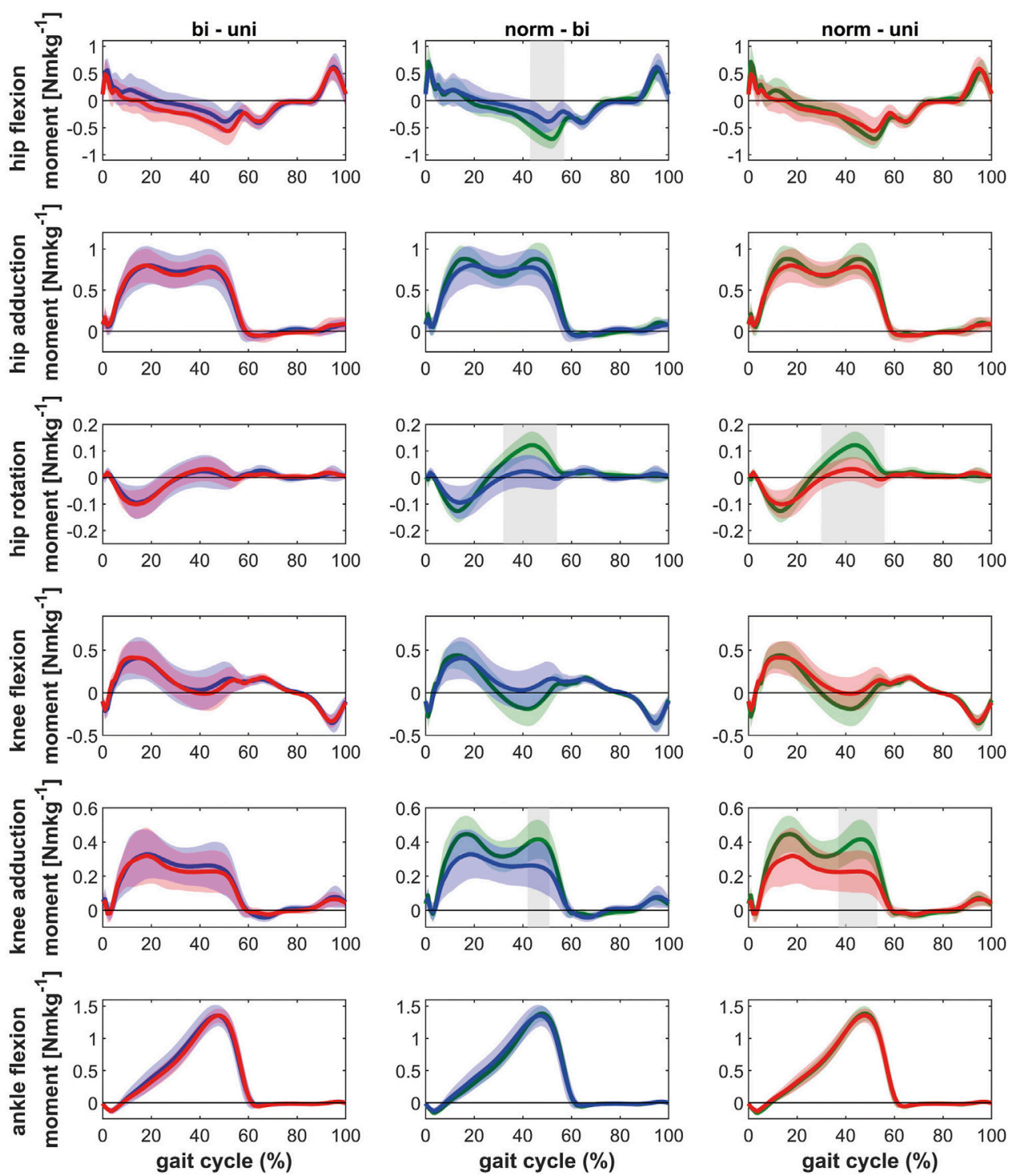

FIGURE 3 | Comparison of the kinetics of the ipsilateral leg between bilateral hip OA patients, unilateral hip OA patients and healthy controls. Mean and one standard deviation of the external joint moments. Shaded areas indicate statistical differences. Blue lines = bilateral patients, red lines $=$ unilateral patients, green lines = healthy controls. (A-C) hip flexion/extension moment: flexion (+)/extension (-); (D-F) hip adduction/abduction moment: adduction (+)/abduction (-); (G-I) hip rotation moment: internal rotation (+)/external rotation (-); (J-L) knee flexion/extension moment: flexion (+)/extension (-); (M-O) knee adduction/abduction moment: varus $(+)$ /valgus (-); (P-R) ankle dorsiflexion/plantarflexion moment: dorsiflexion (+)/plantarflexion (-).

the foot progression angle (not displayed) and the external ankle dorsiflexion/plantarflexion moment (Supplementary Figure 1 and Supplementary Figure 2).

Posthoc tests showed that bilateral patients walked with more thorax lean towards the ipsilateral side, more pelvic tilt and less pelvic obliquity up, less hip extension as well as less knee extension during the stance phase of gait compared to healthy controls (Figure 1). The contralateral leg showed additionally less hip abduction during the swing phase of gait and more dorsiflexion in the ankle joint (Figure 2). The range of motion of both the hip and the knee are limited in the bilateral hip OA patients.
Unilateral patients showed a similar pattern as the bilateral patients for the ipsilateral leg compared to the healthy controls, with more thorax lean towards the ipsilateral side and less hip and knee extension (Figure 1). However also differences were detected: whereas the bilateral patients had less pelvic obliquity up and more pelvic tilt, the unilateral patients showed more thorax tilt and less hip abduction (swing phase). For the contralateral leg, the unilateral patients only showed more thorax tilt and less hip abduction in the swing phase compared to healthy controls (Figure 2).

The ipsilateral leg of bilateral patients did not show any differences compared to the ipsilateral leg of unilateral 

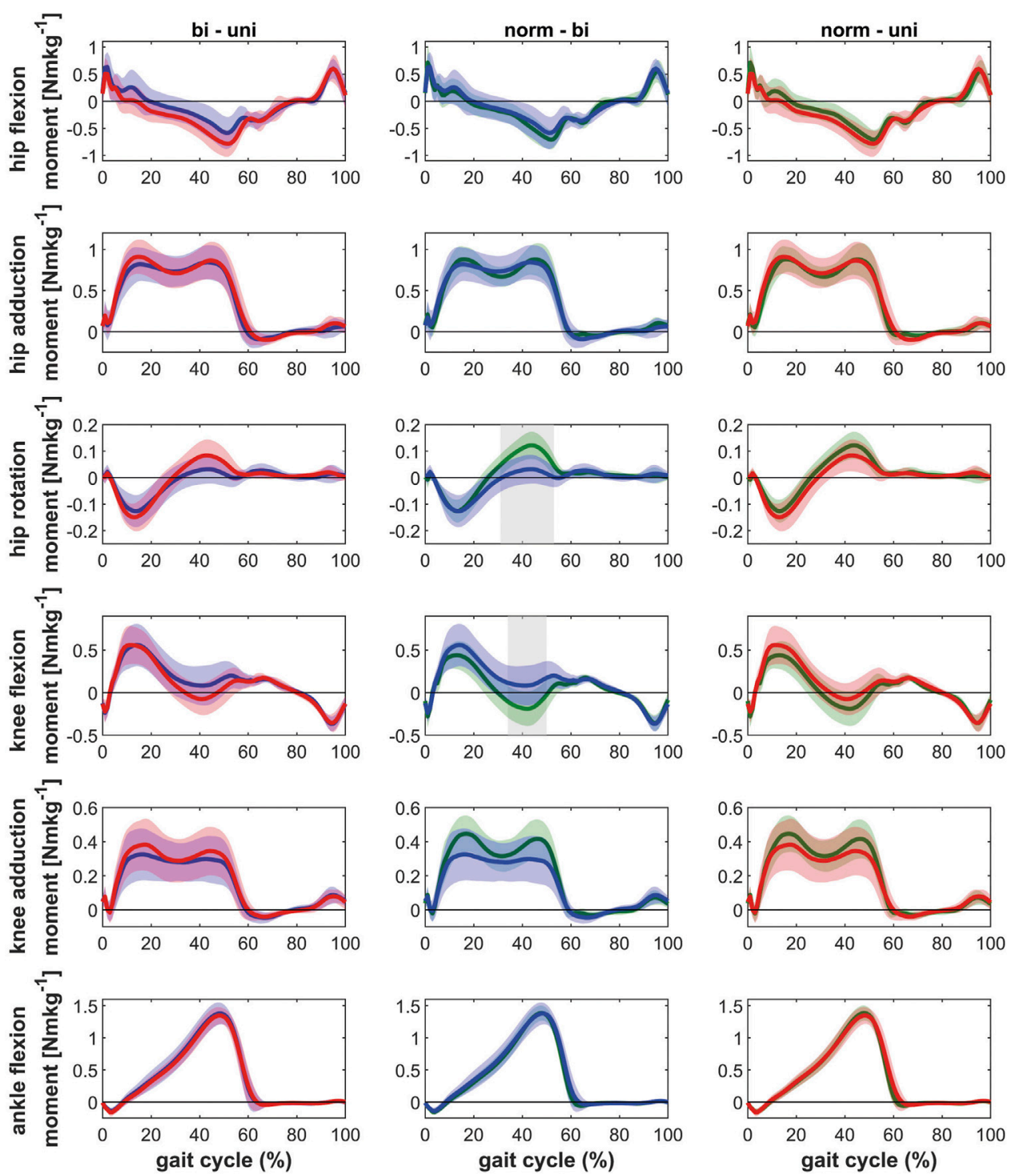

FIGURE 4 | Comparison of the kinetics of the contralateral leg between bilateral hip OA patients, unilateral hip OA patients and healthy controls. Mean and one standard deviation of the external joint moments. Shaded areas indicate statistical differences. Blue lines = bilateral patients, red lines $=$ unilateral patients, green lines = healthy controls. (A-C) hip flexion/extension moment: flexion (+)/extension (-); (D-F) hip adduction/abduction moment: adduction (+)/abduction (-); (G-I) hip rotation moment: internal rotation (+)/external rotation (-); (J-L) knee flexion/extension moment: flexion (+)/extension (-); (M-O) knee adduction/abduction moment: varus $(+)$ /valgus (-); (P-R) ankle dorsiflexion/plantarflexion moment: dorsiflexion (+)/plantarflexion (-).

patients. The contralateral leg of bilateral patients showed less hip and knee extension compared to the contralateral leg of unilateral patients (Figure 1 and Figure 2).

Regarding the kinetic parameters, it was found that the ipsilateral leg of patients with bilateral and unilateral hip OA had lower internal hip rotation moments, as well as a lower external knee adduction moments in the second half of stance compared to healthy controls (Figure 3 and Figure 4). On the ipsilateral side, bilateral patients additionally showed a lower hip extension moment compared to the healthy control group. Only the contralateral leg of bilateral patients showed differences compared to healthy controls: lower internal hip rotation moments and a reduced knee extension moment during the second half of stance.

A closer look at both legs of the bilateral patients showed differences between the ipsilateral leg (leg to be operated on) and the contralateral leg (Figure 5). The most striking differences were the counteracting movements of the thorax and the pelvis in the sagittal plane (tilt) and the reduced hip extension at the end of the stance phase of the ipsilateral leg. The lower hip flexion at the beginning of stance and the lower hip extension at the end of stance defined the reduced hip range of motion in the ipsilateral leg. The reduced range of motion resulted in a slightly lower external hip extension moment in the second half of stance phase for the ipsilateral leg. 

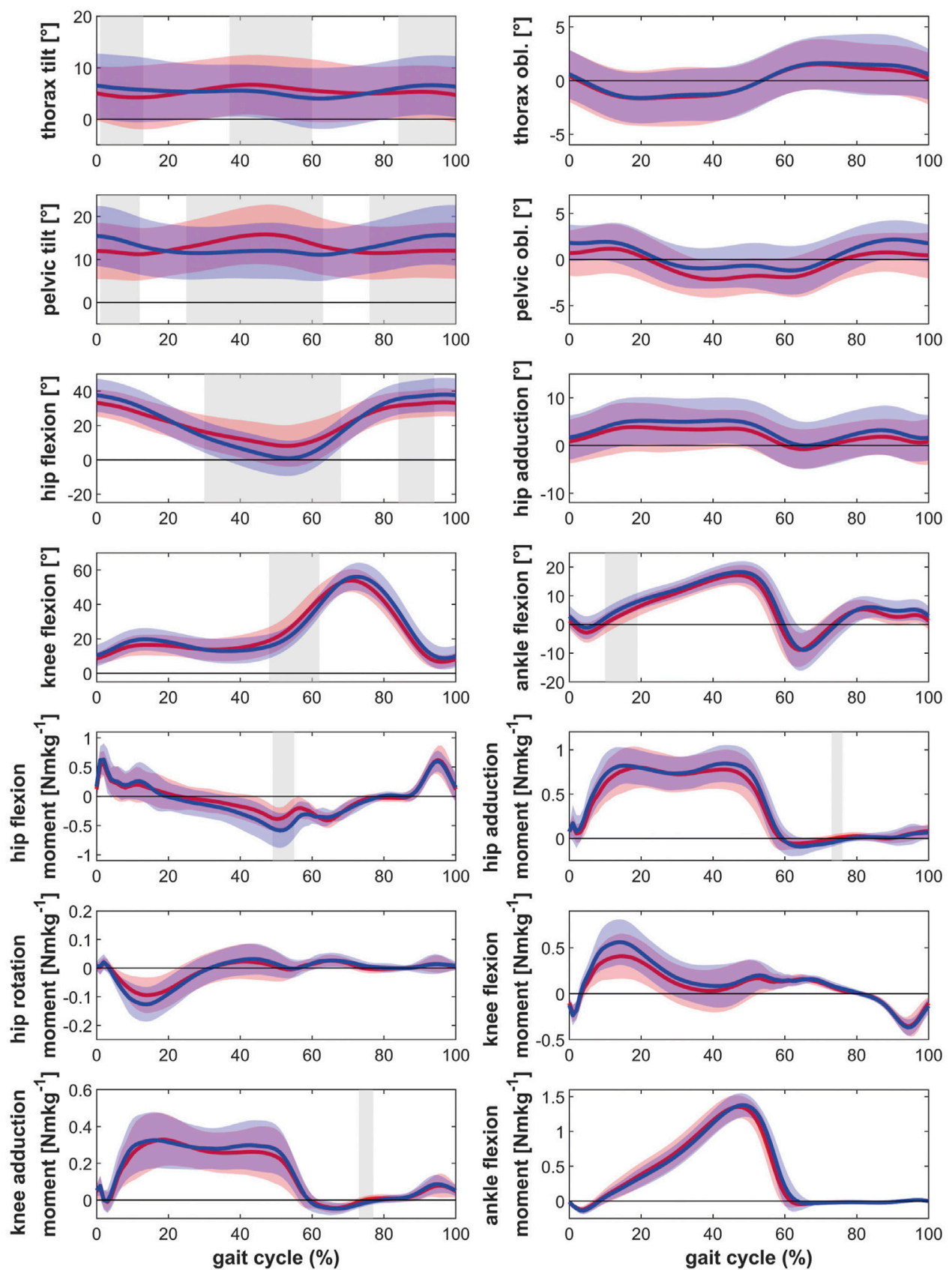

FIGURE 5 | Comparison of the kinematics and kinetics between the ipsilateral and contralateral leg of bilateral hip OA patients. Mean and one standard deviation of the joint angles and the external joint moments. Shaded areas indicate statistical differences. Red lines = ipsilateral leg, blue lines = contralateral leg. (A) thorax tilt: anterior $(+)$ /posterior (-); (B) thorax obliquity: towards the contralateral side (+)/towards the ipsilateral side (-); (C) pelvic tilt: anterior (+)/posterior (-); (D) pelvic obliquity: up $(+) /$ down (-); (E) hip flexion/extension: flexion (+)/extension (-); (F) hip adduction/abduction: adduction (+)/abduction (-); (G) knee flexion/extension: flexion $(+)$ /extension (-); (H) ankle dorsiflexion/plantarflexion: dorsiflexion (+)/plantarflexion (-); (I) hip flexion/extension moment: flexion (+)/extension (-); (J) hip adduction/ abduction moment: adduction (+)/abduction (-); (K) hip rotation moment: internal rotation (+)/external rotation (-); (L) knee flexion/extension moment: flexion $(+)$ /extension (-); (M) knee adduction/abduction moment: varus (+)/valgus (-); (N) ankle dorsiflexion/plantarflexion moment: dorsiflexion (+)/plantarflexion (-).

Besides the above-mentioned differences (thorax tilt, pelvic tilt, hip flexion-extension and hip flexion extension moment), unilateral patients had an asymmetric thorax obliquity (more thorax lean towards the ipsilateral side), higher hip adduction moments during the first half of stance and higher knee adduction moments during the second half of stance in the contralateral leg (Figure 6). The contralateral leg further showed higher rotation moments and more knee extension during the second half of stance compared to the ipsilateral leg. 

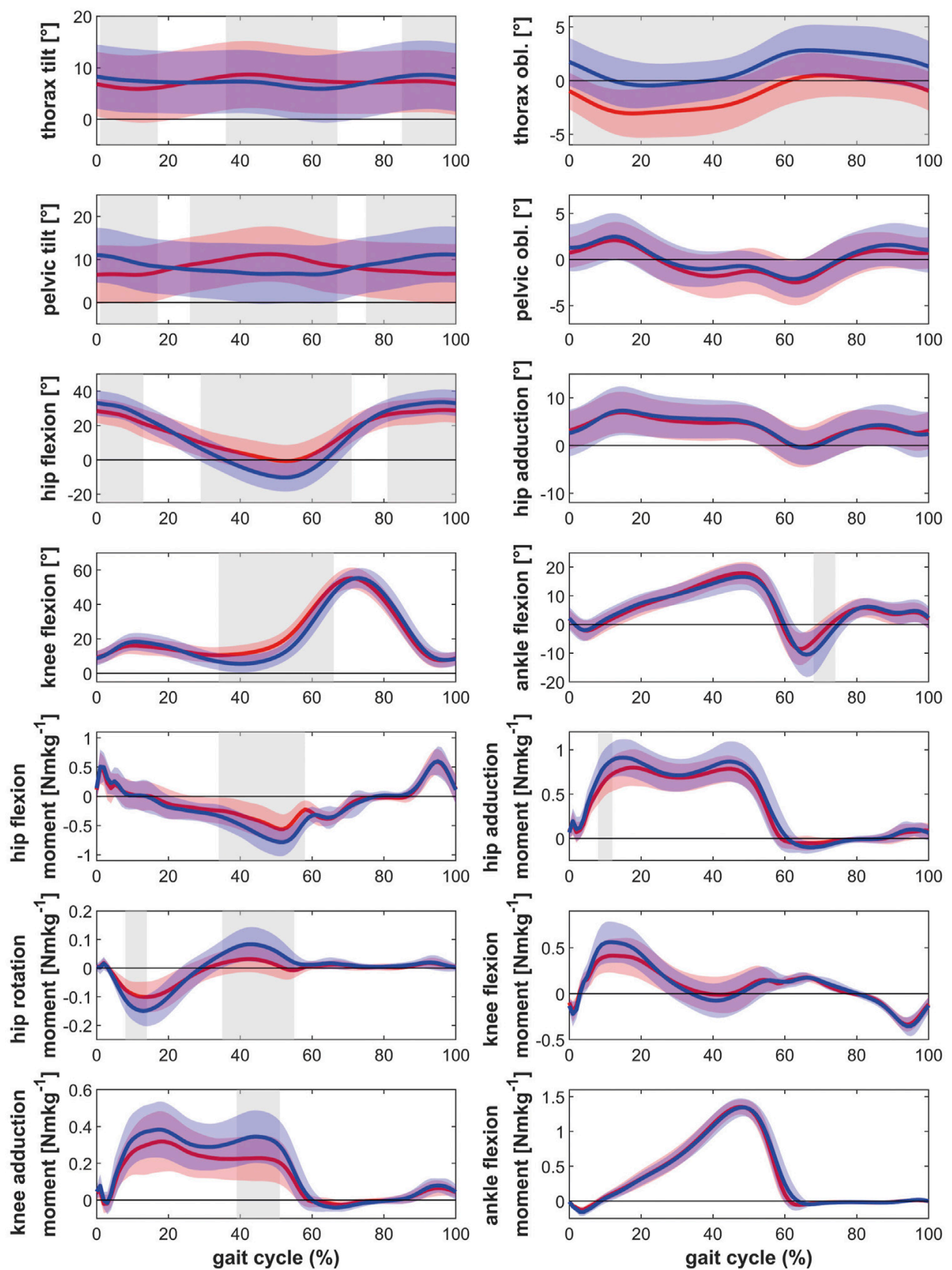

FIGURE 6 | Comparison of the kinematics and kinetics between the ipsilateral and contralateral leg of unilateral hip OA patients. Mean and one standard deviation of the joint angles and the external joint moments. Shaded areas indicate statistical differences. Red lines = ipsilateral leg, blue lines = contralateral leg. (A) thorax tilt: anterior (+)/posterior (-); (B) thorax obliquity: towards the contralateral side (+)/towards the ipsilateral side (-); (C) pelvic tilt: anterior (+)/posterior (-); (D) pelvic obliquity: up (+)/down (-); (E) hip flexion/extension: flexion (+)/extension (-); (F) hip adduction/abduction: adduction (+)/abduction (-); (G) knee flexion/extension: flexion $(+)$ /extension (-); (H) ankle dorsiflexion/plantarflexion: dorsiflexion (+)/plantarflexion (-); (I) hip flexion/extension moment: flexion (+)/extension (-); (J) hip adduction/ abduction moment: adduction (+)/abduction (-); (K) hip rotation moment: internal rotation (+)/external rotation (-); (L) knee flexion/extension moment: flexion $(+)$ /extension (-); (M) knee adduction/abduction moment: varus (+)/valgus (-); (N) ankle dorsiflexion/plantarflexion moment: dorsiflexion (+)/plantarflexion (-).

\section{Symmetry}

The symmetry values differed between bilateral patients and healthy controls only with regard to the hip moment impulse: healthy controls showed a higher gait symmetry compared to the bilateral patients (Figure 7). Unilateral patients showed a lower symmetry compared to healthy controls for the knee adduction moment during the second half of stance, the hip adduction moment during the first half of stance and the hip moment impulse. Only the symmetry in the knee adduction moment during the second half of stance differed significantly between the 


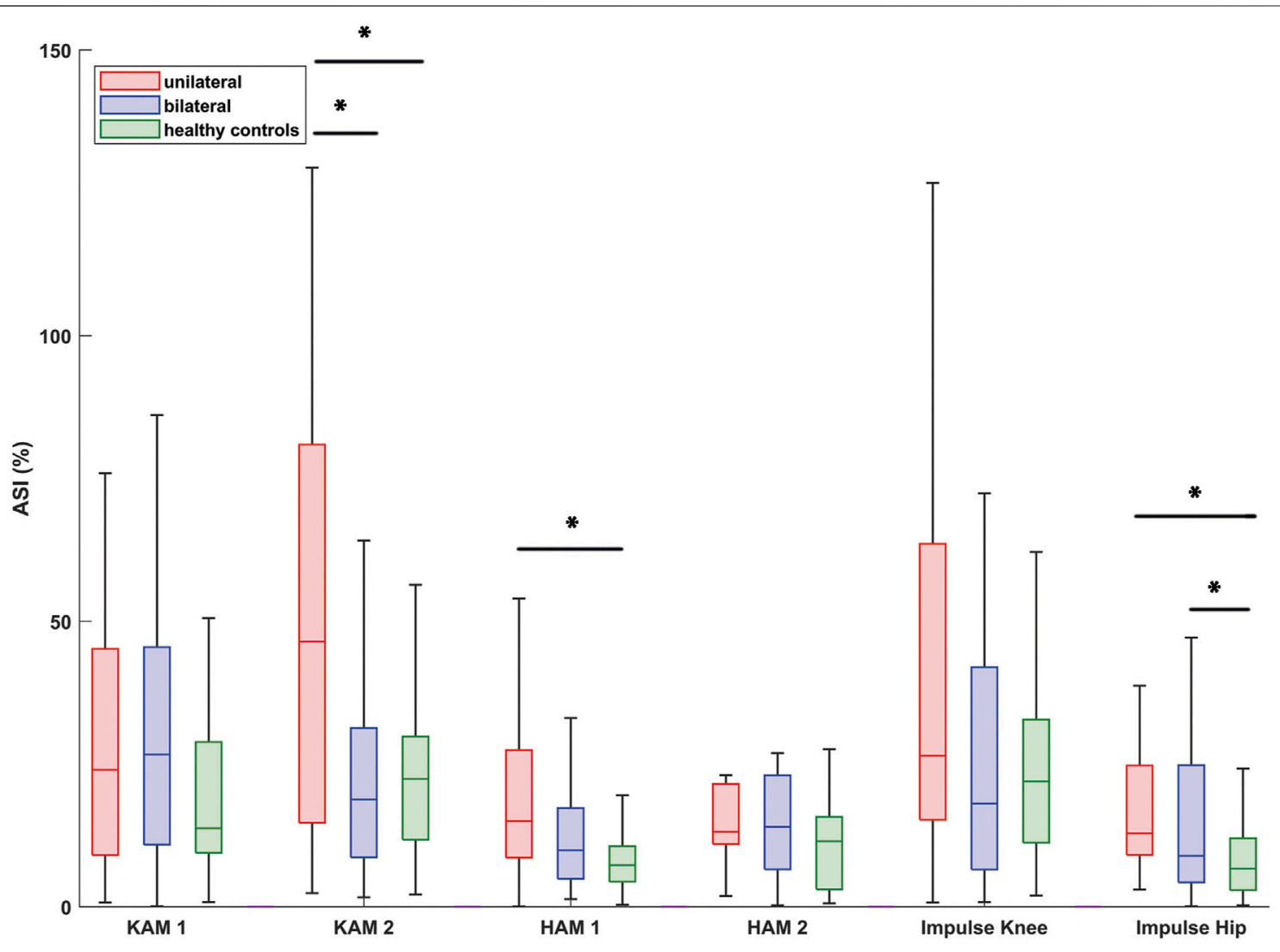

FIGURE 7 | Box-and-whisker-plots of Absolute Symmetry Indices (ASI) of the external knee and hip joint moments as well as the knee and hip impulses. Boxes represent $50 \%$ of the data (interquartile range), the line within the box represents the median and each whisker represents 1.5 times the interquartile range. Significant differences between groups are indicated with an asterisk. Abbreviations: KAM_1/HAM_1: knee / hip adduction moment during the first half of stance; KAM_2 / HAM_2: knee / hip adduction moment during the second half of stance.

bilateral and unilateral patients, with a lower symmetry for unilateral patients.

\section{DISCUSSION}

The main aim of the present study was to investigate the gait pattern of bilateral hip OA patients. This research showed that, in contrast to unilateral patients, the gait pattern of both legs of bilateral patients deviated from matched healthy controls. Although patients with bilateral hip OA show more symmetrical frontal plane knee joint moments, a pathological external knee adduction moment in the second half of stance in the ipsilateral leg is present in patients with unilateral and bilateral hip OA.

\section{Kinematics}

The present study showed that bilateral patients walk with a gait pattern, which has been previously published for unilateral patients: more thorax tilt and lean, more pelvic tilt, less hip extension and less knee extension (Shakoor et al., 2003; Foucher et al., 2007; van Drongelen et al., 2021). The above-mentioned results were found for both legs of the bilateral hip OA patients. Kubota et al. (Kubota et al., 2007) also studied bilateral patients. They found that bilateral patients walked with a forward tilted pelvis and a lean to the ipsilateral side during stance, as was found in the present work. In the current study, significant differences were found between the ipsi- and contralateral leg of bilateral affected OA patients. The patients showed an increased pelvic tilt and a decreased hip extension towards the end of the stance phase for the ipsilateral leg. The contralateral leg had to counter the pelvic movement and showed more hip extension. A comparison to the study of Kubota et al. (Kubota et al., 2007) was not possible, as from that study it did not become clear whether mean values for both legs or data for just one leg were analyzed.

In the present study, a significant higher BMI was found for the unilateral patients compared to the healthy controls. BMI has been associated with lower knee and hip range of motion (Holla et al., 2011) and could be responsible for the differences between the unilateral patients and healthy controls. However, knee and hip range of motion have also been associated with disability (Steultjens et al., 2000) and since no differences were found between bilateral and unilateral patients in BMI nor in the knee and hip flexion the differences in BMI were neglected.

\section{Kinetics}

The second aim of the present study dealt with the magnitude and symmetry of the external knee and hip adduction moments. We 
hypothesized that, in contrast to patients with unilateral hip OA, the external knee and hip adduction moments of the contralateral side are not different compared to the ipsilateral side (more affected side) in bilateral affected patients with hip OA. As a result, bilateral hip OA patients show a more symmetrical joint load compared to unilateral hip OA patients.

For the hip, no differences in the external hip adduction moments were found between patients and healthy controls. This is in contrast to the findings of Foucher et al. (Foucher et al., 2007), who attributed the reduced hip adduction moments on the ipsilateral side in unilateral hip OA patients to an impairment of the hip abductor muscles. Also Kubota et al. (Kubota et al., 2007) reported lower external hip adduction moments. In that study, the reduction in the hip adduction moment was compensated with increased power generation in the ankle joint. However, the patients in the study of Kubota et al. (Kubota et al., 2007) had a very low walking speed $\left(\sim 0.65 \mathrm{~ms}^{-1}\right)$ so that the characteristic double peak was not visible in the hip adduction moment, which might indicate that patients with low functional status were studied (Bahl et al., 2020). The patients in the present study walked at a speed similar to unilateral hip OA patients reported in various studies (Foucher, 2017; Schmidt et al., 2017). In comparison to the healthy controls, the typical double peak in the knee and hip adduction moments seemed to be missing as well (Figures 3,4). However, in the direct comparison of the legs (Figure 5) and in the comparison to unilateral patients it can be seen that the double peak is less pronounced and the curve flattened in patients with bilateral hip OA compared to healthy controls.

Besides the external hip adduction moment, the external knee adduction moments are important parameters (which can be determined by gait analysis), as the peak knee adduction moment has been related to the initiation and progression of knee OA (Miyazaki et al., 2002; Andriacchi and Mündermann, 2006). Both the bilateral and unilateral patients showed a reduced knee adduction moment in the second half of stance phase in the ipsilateral leg, but not in the contralateral leg, compared to the healthy controls. Whereas the unilateral patients showed a clear higher knee adduction moment in the contralateral knee, the bilateral patients had similar knee adduction moments in both knees.

The ipsilateral trunk lean toward the affected stance limb, shown in our patients with unilateral and bilateral hip OA, has been identified as an important compensatory mechanism to unload the hip (Stief et al., 2014). This compensating strategy is also responsible for the redistribution of loads transferred through the medial and lateral knee compartment (Simic et al., 2012; Stief et al., 2014). Body weight and thus the ground reaction force vector is moved toward the center of the knee and hip joint, which reduces medial knee load in a dose-response relationship, with larger lean angles leading to greater reductions in the knee adduction moment (Simic et al., 2012). Mündermann et al. (Mündermann et al., 2008) and van den Noort et al. (van den Noort et al., 2013) have demonstrated that lateral trunk lean has the potential of reducing the knee adduction moment during walking by up to $65 \%$ in healthy subjects. Besides the unloading mechanism, ipsilateral trunk lean can shift the physiological external knee adduction moment into a pathological external abduction moment (valgus moment). This lateral adjustment is argued to increase the lateral tibio-femoral compartment load (Sharma et al., 2000). However, at the moment, it is still questionable whether the change in the medial-to-lateral knee joint load distribution in the ipsilateral leg leads to increased progression or even initiates degenerative changes of the lateral compartment of the knee, since lower medial loading does not necessarily indicate higher lateral loading (van Drongelen et al., 2020b). Various studies reported a higher risk for the development of $\mathrm{OA}$ in the contralateral knee joint after THA (Shakoor et al., 2003; Umeda et al., 2009). However, these studies only reported degenerative findings and did not specify the medial or lateral knee compartment. Gillam et al. (2013) stated that further studies are needed to investigate whether other factors besides load play a role as well. At the moment only Weidow et al. (Weidow et al., 2005) showed an increased probability of $\mathrm{OA}$ in the lateral knee compartment of the ipsilateral leg in patients with hip OA which could be attributed to the morphology of the hip and pelvis in the patient collective.

\section{Symmetry}

We hypothesized that bilateral hip OA patients show a more symmetrical joint load compared to unilateral hip OA patients due to the symmetrical joint moments. Indeed, bilateral hip OA patients walked with more symmetrical knee and hip joint moments, almost similar to healthy controls. Compared to the healthy controls, the bilateral patients only showed lower symmetry in the hip moment impulse. Besides the asymmetry in the hip moment impulse, the unilateral patients also showed a lower symmetry for the hip adduction moment in the first half of stance and the knee adduction moment in the second half of stance compared to the healthy controls.

A closer look at the symmetry data showed that some patients were outside the whiskers, which represent 1.5 times the interquartile range (outliers not displayed in Figure 7). However, in general the gait symmetry of bilateral hip OA patients is comparable to the healthy controls. The present study confirmed the values presented by Schmidt et al. (Schmidt et al., 2017) who found clearly lower symmetry for unilateral hip OA patients compared to healthy controls. Schmidt et al. (Schmidt et al., 2017) reported more variation for unilateral hip OA patients with regard to ASI than healthy controls. Individual adopted gait patterns, which might be caused by pain, can explain the inter-subject variability.

With a more symmetrical load, one could argue that the overload on the contralateral leg is not present in bilateral patients, which could lower the risk for knee and hip OA in the contralateral leg. It must be noted that ASI does not give any qualitative information on which side is overloaded or underloaded. Further, the knee adduction moments in bilateral hip OA patients were lower in both legs compared to healthy controls (significant for the ipsilateral leg and not significant for the contralateral leg). As mentioned above, a lower knee adduction moment might indicate a shift of the load from the medial to the lateral knee compartment (Schmidt et al., 2017), 
which can facilitate degenerative changes of the lateral knee compartment.

A direct comparison of bilateral and unilateral hip OA patients is worth discussing. Usually, bilateral affected patients are excluded from studies on hip OA, which reduces the sample size considerably. From the present study, it became clear that there were no differences in the kinematics and kinetics of the ipsilateral leg between unilateral and bilateral hip OA patients. As such, it can be concluded that the ipsilateral leg of the bilateral patients could be included in studies next to unilateral hip OA patients. In contrast, and based on the larger hip range of motion, the contralateral leg of bilateral patients is not automatically suited for inclusion in studies like the ipsilateral leg.

\section{Limitations}

External knee and hip adduction moments were chosen as the primary outcome, as they are still the most commonly used measures of joint loading with an important relationship to disease progression and initiation (Hurwitz et al., 2001; Miyazaki et al., 2002; Andriacchi and Mündermann, 2006; Lenaerts et al., 2009; Wesseling et al., 2015; Tateuchi et al., 2017). At the moment it is still questionable whether the change in medial-to-lateral knee joint load distribution in the ipsilateral leg leads to increased progression or even initiates degenerative changes of the lateral compartment of the knee, since lower medial loading does not necessarily indicate higher lateral loading (Andriacchi, 2013). Alternative measures of joint load, including compressive force in the medial and lateral knee compartment measured via by musculoskeletal modeling including muscle activity and forces (Holder et al., 2020), might be able to answer this question in the future.

\section{CONCLUSION}

This research showed that, when corrected for speed, the gait kinematics and kinetics of both legs of patients with bilateral hip OA differ from healthy controls. The ipsilateral leg of the bilateral patients could be included in studies in addition to unilateral hip OA patients as no differences were found. Bilateral patients have a symmetrical knee joint loading, in contrast to the asymmetrical knee joint loading in unilateral hip OA patients. Although patients with bilateral hip OA show more symmetrical frontal

\section{REFERENCES}

Adler, R. J., and Taylor, J. E. (2007). Random Fields and Geometry. New York: Springer-Verlag.

Allison, K., Hall, M., Wrigley, T. V., Pua, Y.-H., Metcalf, B., and Bennell, K. L. (2018). Sex-specific Walking Kinematics and Kinetics in Individuals with Unilateral, Symptomatic Hip Osteoarthritis: A Cross Sectional Study. Gait Posture 65, 234-239. doi:10.1016/j.gaitpost.2018.07.179

Andriacchi, T. P. (2013). Editorial: Valgus Alignment and Lateral Compartment Knee Osteoarthritis: A Biomechanical Paradox or New Insight into Knee Osteoarthritis. Arthritis Rheum. 65, 310-313. doi:10.1002/art.37724

Andriacchi, T. P., and Mündermann, A. (2006). The Role of Ambulatory Mechanics in the Initiation and Progression of Knee Osteoarthritis. Curr. Opin. Rheumatol. 18, 514-518. doi:10.1097/01.bor.0000240365.16842.4e plane knee joint moments, a pathological external knee adduction moment in the second half of stance in the ipsilateral leg is present in both patients with unilateral and bilateral hip OA.

\section{DATA AVAILABILITY STATEMENT}

The raw data supporting the conclusion of this article will be made available by the authors, without undue reservation.

\section{ETHICS STATEMENT}

The studies involving human participants were reviewed and approved by the Medical Ethics Committee of the Faculty of Medicine of the Goethe University Frankfurt. The patients/ participants provided their written informed consent to participate in this study.

\section{AUTHOR CONTRIBUTIONS}

$\mathrm{SD}, \mathrm{FS}$, and AM were involved in the design of the study. SD, SB, and FS carried out the data analysis. SD, SB, AM, and FS were involved in the interpretation and discussion of the results. SD took the lead in writing the manuscript. All authors provided critical feedback and contributed to the final manuscript.

\section{ACKNOWLEDGMENTS}

The authors would like to thank Hanna Kaldowski, André Schmidt and Anna Schäfer for their help with the data collection and Jana Holder for her help with the preparation of the manuscript.

\section{SUPPLEMENTARY MATERIAL}

The Supplementary Material for this article can be found online at: https://www.frontiersin.org/articles/10.3389/fbioe.2021.756460/ full\#supplementary-material

Bahl, J. S., Arnold, J. B., Taylor, M., Solomon, L. B., and Thewlis, D. (2020). Lower Functioning Patients Demonstrate Atypical Hip Joint Loading before and Following Total Hip Arthroplasty for Osteoarthritis. J. Orthop. Res. 38, 1550-1558. doi:10.1002/jor.24716

Baker, R., Leboeuf, F., Reay, J., and Sangeux, M. (2018). "The Conventional Gait Model - success and Limitations," in Handbook of Human Motion. Editors B. Müller and S. I. Wolf (New York: Springer International Publishing), 1-19. doi:10.1007/978-3-319-14418-4_25

Berman, A. T., Quinn, R. H., and Zarro, V. J. (1991). Quantitative Gait Analysis in Unilateral and Bilateral Total Hip Replacements. Arch. Phys. Med. Rehabil. 72, 190-194.

Blasimann, A., Dauphinee, S. W., and Staal, J. B. (2014). Translation, CrossCultural Adaptation, and Psychometric Properties of the German Version of the Hip Disability and Osteoarthritis Outcome Score. J. Orthop. Sports Phys. Ther. 44, 989-997. doi:10.2519/jospt.2014.4994 
Bosch, K., and Rosenbaum, D. (2010). Gait Symmetry Improves in Childhood-A 4year Follow-Up of Foot Loading Data. Gait Posture 32, 464-468. doi:10.1016/ j.gaitpost.2010.07.002

Davis, R. B., Ôunpuu, S., Tyburski, D., and Gage, J. R. (1991). A Gait Analysis Data Collection and Reduction Technique. Hum. Mov. Sci. 10, 575-587. doi:10.1016/ 0167-9457(91)90046-Z

Foucher, K. C., Hurwitz, D. E., and Wimmer, M. A. (2007). Preoperative Gait Adaptations Persist One Year after Surgery in Clinically Well-Functioning Total Hip Replacement Patients. J. Biomech. 40, 3432-3437. doi:10.1016/ j.jbiomech.2007.05.020

Foucher, K. C. (2017). Sex-specific Hip Osteoarthritis-Associated Gait Abnormalities: Alterations in Dynamic Hip Abductor Function Differ in Men and Women. Clin. Biomech. 48, 24-29. doi:10.1016/ j.clinbiomech.2017.07.002

Gillam, M. H., Lie, S. A., Salter, A., Furnes, O., Graves, S. E., Havelin, L. I., et al. (2013). The Progression of End-Stage Osteoarthritis: Analysis of Data from the Australian and Norwegian Joint Replacement Registries Using a Multi-State Model. Osteoarthr. Cartil. 21, 405-412. doi:10.1016/j.joca.2012.12.008

Günther, K. P., Stürmer, T., Sauerland, S., Zeissig, I., Sun, Y., Kessler, S., et al. (1998). Prevalence of Generalised Osteoarthritis in Patients with Advanced Hip and Knee Osteoarthritis: the Ulm Osteoarthritis Study. Ann. Rheum. Dis. 57, 717-723. doi:10.1136/ard.57.12.717

Harris, W. H. (1969). Traumatic Arthritis of the Hip after Dislocation and Acetabular Fractures. J. Bone Joint Surg. Am. 51, 737-755. doi:10.2106/ 00004623-196951040-00012

Herger, S., Vach, W., Liphardt, A.-M., Egloff, C., Nüesch, C., and Mündermann, A. (2019). Dose-response Relationship between Ambulatory Load Magnitude and Load-Induced Changes in COMP in Young Healthy Adults. Osteoarthr. Cartil. 27, 106-113. doi:10.1016/j.joca.2018.09.002

Holder, J., Trinler, U., Meurer, A., and Stief, F. (2020). A Systematic Review of the Associations between Inverse Dynamics and Musculoskeletal Modeling to Investigate Joint Loading in a Clinical Environment. Front. Bioeng. Biotechnol. 8, 603907. doi:10.3389/fbioe.2020.603907

Holla, J. F. M., Steultjens, M. P. M., van der Leeden, M., Roorda, L. D., BiermaZeinstra, S. M. A., den Broeder, A. A., et al. (2011). Determinants of Range of Joint Motion in Patients with Early Symptomatic Osteoarthritis of the Hip And/ or Knee: an Exploratory Study in the CHECK Cohort. Osteoarthr. Cartil. 19, 411-419. doi:10.1016/j.joca.2011.01.013

Hurwitz, D. E., Sumner, D. R., and Block, J. A. (2001). Bone Density, Dynamic Joint Loading and Joint Degeneration. Cells Tissues Organs 169, 201-209. doi:10.1159/000047883

Joseph, G. B., Hilton, J. F., Jungmann, P. M., Lynch, J. A., Lane, N. E., Liu, F., et al. (2016). Do persons with Asymmetric Hip Pain or Radiographic Hip OA Have Worse Pain and Structure Outcomes in the Knee Opposite the More Affected Hip? Data from the Osteoarthritis Initiative. Osteoarthr. Cartil. 24, 427-435. doi:10.1016/j.joca.2015.10.001

Jungmann, P. M., Nevitt, M. C., Baum, T., Liebl, H., Nardo, L., Liu, F., et al. (2015). Relationship of Unilateral Total Hip Arthroplasty (THA) to Contralateral and Ipsilateral Knee Joint Degeneration - a Longitudinal 3T MRI Study from the Osteoarthritis Initiative (OAI). Osteoarthr. Cartil. 23, 1144-1153. doi:10.1016/ j.joca.2015.03.022

Kadaba, M. P., Ramakrishnan, H. K., and Wootten, M. E. (1990). Measurement of Lower Extremity Kinematics during Level Walking. J. Orthop. Res. 8, 383-392. doi:10.1002/jor.1100080310

Kellgren, J. H., and Lawrence, J. S. (1957). Radiological Assessment of OsteoArthrosis. Ann. Rheum. Dis. 16, 494-502. doi:10.1136/ard.16.4.494

Kubota, M., Shimada, S., Kobayashi, S., Sasaki, S., Kitade, I., Matsumura, M., et al. (2007). Quantitative Gait Analysis of Patients with Bilateral Hip Osteoarthritis Excluding the Influence of Walking Speed. J. Orthop. Sci. 12, 451-457. doi:10.1007/s00776-007-1160-z

Lenaerts, G., Mulier, M., Spaepen, A., Van der Perre, G., and Jonkers, I. (2009). Aberrant Pelvis and Hip Kinematics Impair Hip Loading before and after Total Hip Replacement. Gait Posture 30, 296-302. doi:10.1016/ j.gaitpost.2009.05.016

Miyazaki, T., Wada, M., Kawahara, H., Sato, M., Baba, H., and Shimada, S. (2002). Dynamic Load at Baseline Can Predict Radiographic Disease Progression in Medial Compartment Knee Osteoarthritis. Ann. Rheum. Dis. 61, 617-622. doi:10.1136/ard.61.7.617
Morcos, M. W., Hart, A., Antoniou, J., Huk, O. L., Zukor, D. J., and Bergeron, S. G. (2018). No Difference in Major Complication and Readmission Rates Following Simultaneous Bilateral vs Unilateral Total Hip Arthroplasty. J. Arthroplasty 33, 2541-2545. doi:10.1016/j.arth.2018.03.050

Mündermann, A., Asay, J. L., Mündermann, L., and Andriacchi, T. P. (2008). Implications of Increased Medio-Lateral Trunk Sway for Ambulatory Mechanics. J. Biomech. 41, 165-170. doi:10.1016/j.jbiomech.2007.07.001

Nilsdotter, A. K., Lohmander, L. S., Klässbo, M., and Roos, E. M. (2003). Hip Disability and Osteoarthritis Outcome Score (HOOS) - Validity and Responsiveness in Total Hip Replacement. BMC Musculoskelet. Disord. 4, 10. doi:10.1186/1471-2474-4-10

Pataky, T. C., Robinson, M. A., and Vanrenterghem, J. (2013). Vector Field Statistical Analysis of Kinematic and Force Trajectories. J. Biomech. 46, 2394-2401. doi:10.1016/j.jbiomech.2013.07.031

Robinson, R. O., Herzog, W., and Nigg, B. M. (1987). Use of Force Platform Variables to Quantify the Effects of Chiropractic Manipulation on Gait Symmetry. J. Manipulative Physiol. Ther. 10, 172-176.

Santana, D. C., Anis, H. K., Mont, M. A., Higuera, C. A., and Piuzzi, N. S. (2020). What Is the Likelihood of Subsequent Arthroplasties after Primary TKA or THA? Data from the Osteoarthritis Initiative. Clin. Orthop. Relat. Res. 478, 34-41. doi:10.1097/corr.0000000000000925

Schmidt, A., Meurer, A., Lenarz, K., Vogt, L., Froemel, D., Lutz, F., et al. (2017). Unilateral Hip Osteoarthritis: The Effect of Compensation Strategies and Anatomic Measurements on Frontal Plane Joint Loading. J. Orthop. Res. 35, 1764-1773. doi:10.1002/jor.23444

Shakoor, N., Dua, A., Thorp, L. E., Mikolaitis, R. A., Wimmer, M. A., Foucher, K. C., et al. (2011). Asymmetric Loading and Bone mineral Density at the Asymptomatic Knees of Patients with Unilateral Hip Osteoarthritis. Arthritis Rheum. 63, 3853-3858. doi:10.1002/art.30626

Shakoor, N., Hurwitz, D. E., Block, J. A., Shott, S., and Case, J. P. (2003). Asymmetric Knee Loading in Advanced Unilateral Hip Osteoarthritis. Arthritis Rheum. 48, 1556-1561. doi:10.1002/art.11034

Sharma, L., Lou, C., Cahue, S., and Dunlop, D. D. (2000). The Mechanism of the Effect of Obesity in Knee Osteoarthritis: the Mediating Role of Malalignment. Arthritis Rheum. 43, 568-575. doi:10.1002/1529-0131(200003)43:3<568:aidanr13>3.0.co;2-e

Simic, M., Hunt, M. A., Bennell, K. L., Hinman, R. S., and Wrigley, T. V. (2012). Trunk Lean Gait Modification and Knee Joint Load in People with Medial Knee Osteoarthritis: the Effect of Varying Trunk Lean Angles. Arthritis Care Res. 64, 1545-1553. doi:10.1002/acr.21724

Solomonow-Avnon, D., Haim, A., Levin, D., Elboim-Gabyzon, M., Rozen, N., Peled, E., et al. (2016). Reduction of Hip Joint Reaction Force via Medio-Lateral Foot center of Pressure Manipulation in Bilateral Hip Osteoarthritis Patients. J. Orthop. Res. 34, 1762-1771. doi:10.1002/jor.23190

Steultjens, M. P. M., Dekker, J., van Baar, M. E., Oostendorp, R. A. B., and Bijlsma, J. W. J. (2000). Range of Joint Motion and Disability in Patients with Osteoarthritis of the Knee or Hip. Rheumatology 39, 955-961. doi:10.1093/ rheumatology/39.9.955

Stief, F., Böhm, H., Ebert, C., Döderlein, L., and Meurer, A. (2014). Effect of Compensatory Trunk Movements on Knee and Hip Joint Loading during Gait in Children with Different Orthopedic Pathologies. Gait Posture 39, 859-864. doi:10.1016/j.gaitpost.2013.11.012

Stief, F., Böhm, H., Michel, K., Schwirtz, A., and Döderlein, L. (2013). Reliability and Accuracy in Three-Dimensional Gait Analysis: a Comparison of Two Lower Body Protocols. J. Appl. Biomech. 29, 105-111. doi:10.1123/jab.29.1.105

Stief, F., Schmidt, A., van Drongelen, S., Lenarz, K., Froemel, D., Tarhan, T., et al. (2018). Abnormal Loading of the Hip and Knee Joints in Unilateral Hip Osteoarthritis Persists Two Years after Total Hip Replacement. J. Orthop. Res. 36, 2167-2177. doi:10.1002/JOR.23886

Tateuchi, H., Koyama, Y., Akiyama, H., Goto, K., So, K., Kuroda, Y., et al. (2017). Daily Cumulative Hip Moment Is Associated with Radiographic Progression of Secondary Hip Osteoarthritis. Osteoarthr. Cartil. 25, 1291-1298. doi:10.1016/ j.joca.2017.02.796

Umeda, N., Miki, H., Nishii, T., Yoshikawa, H., and Sugano, N. (2009). Progression of Osteoarthritis of the Knee After Unilateral Total Hip Arthroplasty: Minimum 10-year Follow-up Study. Arch. Orthop. Trauma. Surg 129, 149-154. doi:10.1007/s00402-008-0577-y 
van den Noort, J. C., Schaffers, I., Snijders, J., and Harlaar, J. (2013). The Effectiveness of Voluntary Modifications of Gait Pattern to Reduce the Knee Adduction Moment. Hum. Mov. Sci. 32, 412-424. doi:10.1016/ j.humov.2012.02.009

van Drongelen, S., Kaldowski, H., Fey, B., Tarhan, T., Assi, A., Stief, F., et al. (2020a). Determination of Leg Alignment in Hip Osteoarthritis Patients with the EOS $^{\circledR}$ System and the Effect on External Joint Moments during Gait. Appl. Sci. 10, 7777. doi:10.3390/app10217777

van Drongelen, S., Stetter, B. J., Böhm, H., Stief, F., Stein, T., and Meurer, A. (2021). Identification of Patients with Similar Gait Compensating Strategies Due to Unilateral Hip Osteoarthritis and the Effect of Total Hip Replacement: A Secondary Analysis. J. Clin. Med. 10, 2167. doi:10.3390/jcm10102167

van Drongelen, S., Wesseling, M., Holder, J., Meurer, A., and Stief, F. (2020b). Knee Load Distribution in Hip Osteoarthritis Patients after Total Hip Replacement. Front. Bioeng. Biotechnol. 8. doi:10.3389/fbioe.2020.578030

Weidow, J., Mars, I., and Kärrholm, J. (2005). Medial and Lateral Osteoarthritis of the Knee is Related to Variations of Hip and Pelvic Anatomy. Osteoarthr. Cartil. 13, 471-477. doi:10.1016/j.joca.2005.01.009

Wesseling, M., de Groote, F., Meyer, C., Corten, K., Simon, J.-P., Desloovere, K., et al. (2015). Gait Alterations to Effectively Reduce Hip Contact Forces. J. Orthop. Res. 33, 1094-1102. doi:10.1002/jor.22852

Wesseling, M., Meyer, C., Corten, K., Desloovere, K., and Jonkers, I. (2018). Longitudinal Joint Loading in Patients before and up to One Year after
Unilateral Total Hip Arthroplasty. Gait Posture 61, 117-124. doi:10.1016/ j.gaitpost.2018.01.002

Woltring, H. J. (1986). A Fortran Package for Generalized, Cross-Validatory Spline Smoothing and Differentiation. Adv. Eng. Softw. (1978) 8, 104-113. doi:10.1016/0141-1195(86)90098-7

Conflict of Interest: The authors declare that the research was conducted in the absence of any commercial or financial relationships that could be construed as a potential conflict of interest.

Publisher's Note: All claims expressed in this article are solely those of the authors and do not necessarily represent those of their affiliated organizations, or those of the publisher, the editors and the reviewers. Any product that may be evaluated in this article, or claim that may be made by its manufacturer, is not guaranteed or endorsed by the publisher.

Copyright (C) 2021 van Drongelen, Braun, Stief and Meurer. This is an open-access article distributed under the terms of the Creative Commons Attribution License (CC $B Y)$. The use, distribution or reproduction in other forums is permitted, provided the original author(s) and the copyright owner(s) are credited and that the original publication in this journal is cited, in accordance with accepted academic practice. No use, distribution or reproduction is permitted which does not comply with these terms. 